Proceedings of the 26th Annual International Conference of the IEEE EMBS

San Francisco, CA, USA • September 1-5, 2004

\title{
VALIDATION OF A COMPUTER-AIDED EMG DECOMPOSITION METHOD
}

\author{
Kevin C. McGill, Zoia C. Lateva, M. Elise Johanson \\ Rehabilitation R\&D Center \\ VA Palo Alto Health Care System \\ Palo Alto, CA, USA; email: mcgill@ rrdmail.stanford.edu
}

\begin{abstract}
This paper presents an objective assessment of the accuracy of EMGLAB, a computer-aided EMG decomposition program that we developed. EMG signals were recorded simultaneously using monopolar needle and fine-wire electrodes from nearby sites in the tibialis anterior muscle during moderate isometric contractions. The signals were decomposed independently by an experienced operator, yielding 3-12 (mean 8.7) motor-unit action potential (MUAP) trains per signal. Decomposition accuracy was estimated by crosschecking 83 pairs of trains from different signals that corresponded to the same motor units. The results show that EMGLAB was able to decompose large MUAPs (peak amplitudes greater than 2.5 times rms signal amplitude) with 98-100\% accuracy, and smaller MUAPs with $80-100 \%$ accuracy. Many of the errors involved misalignment of small MUAPs within superimpositions and amounted to less than $5 \mathrm{~ms}$. These results validate the accuracy of EMGLAB for decomposing EMG signals of moderate complexity.
\end{abstract}

\section{INTRODUCTION}

The electromyographic (EMG) signal is made up of trains of discharges called motor-unit action potentials (MUAPs). Decomposing EMG signals into their constituent MUAP trains is of interest for assessing motor-unit (MU) organization and behavior in experimental and clinical neurophysiology $[1,2,7]$. EMGLAB is a computer-aided method that we developed to fully decompose moderately complex EMG signals. We have used EMGLAB extensively to study MU architecture [4,5]. The purpose of this paper is to access the accuracy of EMGLAB.

Assessing the accuracy of an EMG decomposition requires an independent way to ascertain the true composition of the signal. One approach is to use simulated signals of known composition [3]. However, this approach is only valid to the extent that all the factors that effect decomposition accuracy are known and can be realistically simulated.

This work was funded by the Medical and Rehabilitation R\&D Services of the US Department of Veterans Affairs and by the US National Institutes of Health (R01 AR049894).
A second approach is to use pairs of actual signals recorded simultaneously from nearby sites in the same muscle [2]. Such signals will contain activity from some of the same MUs, and so can be used to cross check each other. Where the decompositions disagree, at least one of them must be in error. But where they agree, they are very likely both to be correct. (Otherwise they would both have to be in error by precisely the same amount, which is unlikely given the different characteristics of the different signals.) This approach thus provides an objective way to assess decomposition accuracy using actual signals.

In this paper we use the cross-checking approach to access the accuracy of EMGLAB.

\section{METHODS}

\subsection{EMG Recording}

EMG signals were recorded simultaneously using two monopolar fine-wire electrodes and a monopolar needle electrode in the tibialis anterior muscle of one normal subject. The wire electrodes were $50 \mu \mathrm{m}$ in diameter and insulated except for $1 \mathrm{~mm}$ at the tip. They were inserted together using a single hypodermic needle, with their lead-off surfaces separated by about $2 \mathrm{~mm}$ due to different barb lengths. The needle electrode was inserted at different locations and different depths within $10 \mathrm{~mm}$ distal or proximal to the wires. Signals were recorded during a total of 710 -s moderate isometric contractions. The signals were amplified with $5 \mathrm{~Hz}-$ $5 \mathrm{kHz}$ filter settings and sampled at $10 \mathrm{kHz}$. The study was approved by the Stanford University Committee on the Use of Human Subjects in Research, and the subject gave informed consent.

\subsection{Decomposition}

Each signal was decomposed independently by an experienced operator using EMGLAB. EMGLAB is an interactive program written in Mablab for EMG decomposition. It uses a graphical user interface with four panels that show a segment of the EMG signal, the templates of the identified 


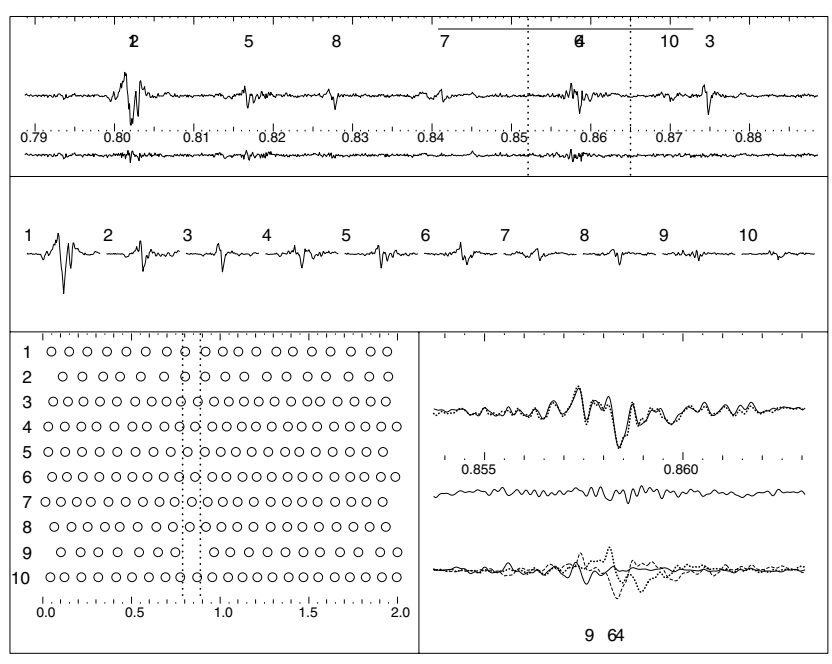

Figure 1. EMGLAB computer screen. Top: Signal panel, showing a segment of the EMG signal (high-pass filtered at $1 \mathrm{kHz}$, top trace) and the residual after template matching (bottom trace). The numbers indicate the identified MU discharges. The horizontal line indicates the expected discharge time of MU 9. Middle: Template panel, showing the templates of the identified MUs. Lower left: Firing-pattern panel, showing the identified discharge times of each MU. Lower right: Close-up panel, showing a superimposition at an expanded scale (top dotted trace), a configuration of templates (bottom traces and numbers), the sum of the templates (top solid trace), and the residual (center trace). Time scales are in seconds. Except for one discharge of MU 9, which has been identified in the close-up panel but not yet updated to the other panels, this decomposition would be judged accurate and complete because of the small amplitude of the residual signal and the regularity of the firing patterns.

MUAPs, the firing patterns of the identified MUAP trains, and a close-up of the signal for resolving superimpositions (see Fig. 1).

The EMG signals were digitally high-pass filtered at $1 \mathrm{kHz}$. The operator used a combination of automatic and manual methods to form templates of the active MUs and identify their discharges in the signal. EMGLAB includes sophisticated algorithms for aligning templates and resolving superimpositions [6], and it uses interpolation to achieve a temporal resolution of $\pm 0.01 \mathrm{~ms}$. The operator judged the accuracy and completeness of each decomposition by assessing the residual that remained after the templates of the identified MUs were subtracted from the signal and by assessing the regularity and completeness of the identified firing patterns.
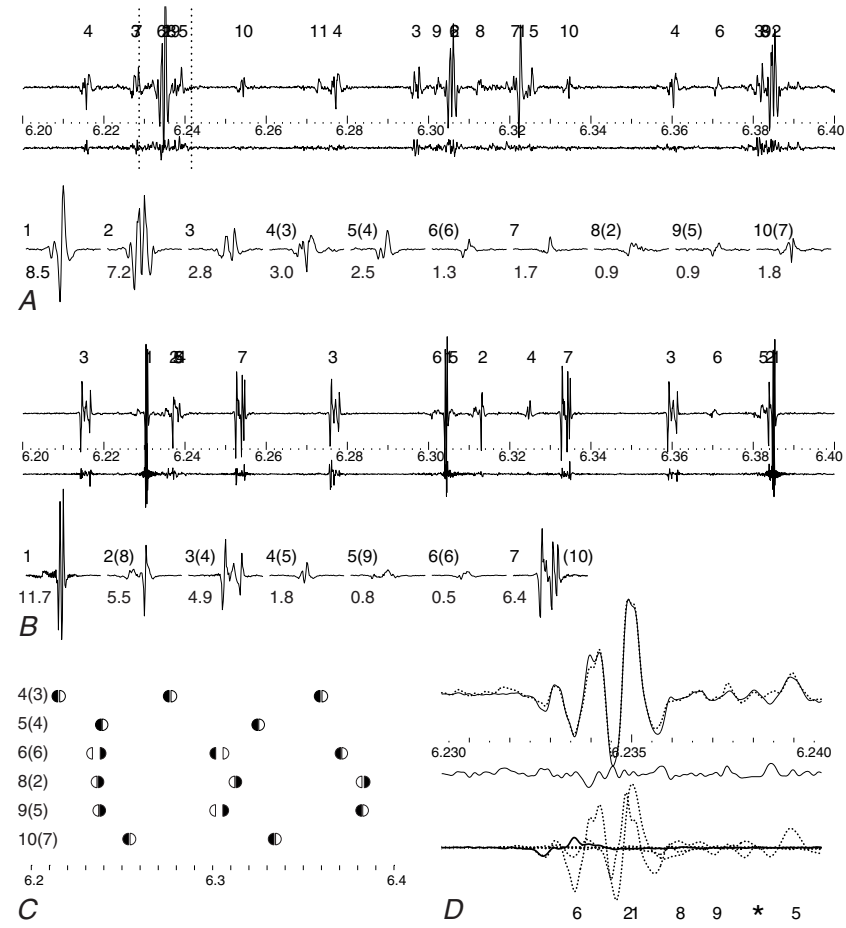

Figure 2. Cross checking decomposition results. $(A, B)$ Segments of decomposition results of two signals from nearby recording sites. Six MUs were detected in both signals, as indicated by the numbers in parentheses. For example, MU 4 in A was the same as MU 3 in B. The SNRs of the templates are shown. $(C)$ Discharge times as identified in A (open half circles) and B (filled half circles). Discrepancies of about $4 \mathrm{~ms}$ can be seen for the first two discharges of MU 6(6) and the second discharge of MU 9(5). Inspection shows that decomposition B was probably correct in all three cases. $(D)$ Close-up of the superimposition at $6.235 \mathrm{~s}$ in A. The asterisk shows the correct location of MU 6 according to decomposition B.

\subsection{Decomposition Accuracy}

MUAP trains in different signals from the same contraction were considered to correspond to the same MU if at least $50 \%$ of their discharges agreed to within $\pm 5 \mathrm{~ms}$. The discharge times of one train were adjusted by the median of the between-signal offset to compensate for any conduction delay between the two recording sites. Then the percentage of discharges for which the two decompositions agreed was determined at two levels of precision: \pm 0.5 and $\pm 5 \mathrm{~ms}$. In order to compare MUAPs in signals with different levels of activity and background noise, each MUAP was characterized by its signal-to-noise ratio (SNR), equal to its peak amplitude divided by the rms signal amplitude. 


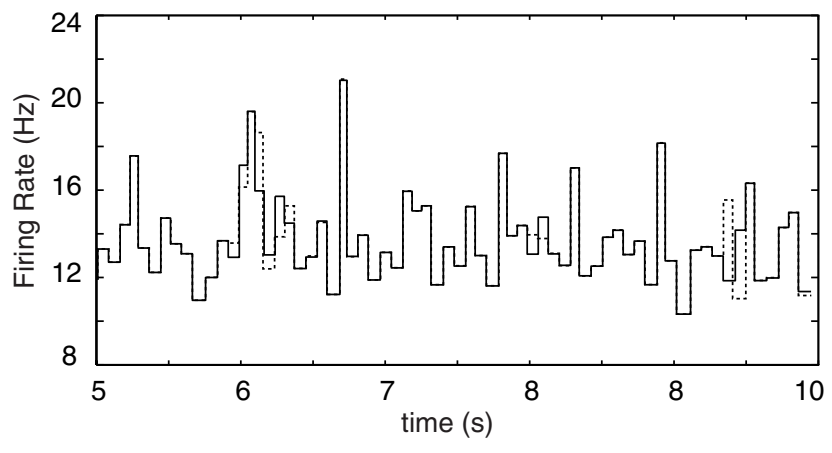

Figure 3. Instantaneous firing rate of MU 6(6) from Fig. 2 determined by decomposition A (dashed) and decomposition B (solid). The discrepancies between the two decompositions resulted in only minor discrepancies in the overall firing patterns.

\section{RESULTS}

An example is shown in Fig. 2. Signal A was recorded by one of the fine-wire electrodes, and signal B by the needle electrode. Six of the MUs identified in signal A were also identified in signal B. In the segment shown, both decompositions agreed to within $\pm 0.5 \mathrm{~ms}$ on the timings of all but three of the discharges of the common MUs. They disagreed by about $4 \mathrm{~ms}$ on the first and second discharges of MUAP 6(6) and the second discharge of MUAP 9(5). Over the entire 10-s record, the two decompositions disagreed on 10 of the 135 discharges of MUAP 6(6) (7\%), only 4 of these disagreements $(3 \%)$ being by more than $5 \mathrm{~ms}$. The overall firing patterns determined by the two decompositions were largely identical (Fig. 3).

Overall, the number of MUs identified per signal ranged from 3 to 12 (mean 8.7). A total of 83 pairs of MUAP trains that corresponded to the same MU were found. The agreement rates are plotted in Fig. 4. Although disagreements can be due to decomposition errors in either signal, a conservative estimate is to consider them reflective of the error rate in the signal in which the MU had the smaller SNR. Therefore the points in Fig. 4 are plotted against the SNR of the smaller MUAP. The rates for \pm 0.5 -ms agreement ranged from $83 \%$ for the smallest MUAPs $(\mathrm{SNR}=0.5)$ to $98-100 \%$ for large ones (SNR $>2.5$ ). $50 \%$ of the disagreements were less than $5 \mathrm{~ms}$, and all but 10 of the \pm 5 -ms agreement rates were above $95 \%$.

An analysis of the discrepancies revealed three common types of decomposition errors: incorrect location of small MUAPs within a superimposition (such as MU 6 in Fig. 2D), assignment of small MUAPs to the incorrect superimposition, and confusion of MUAPs with similar shapes (such as MUs 6 and 9 near $6.35 \mathrm{~s}$ in Fig. 2A). Errors due to
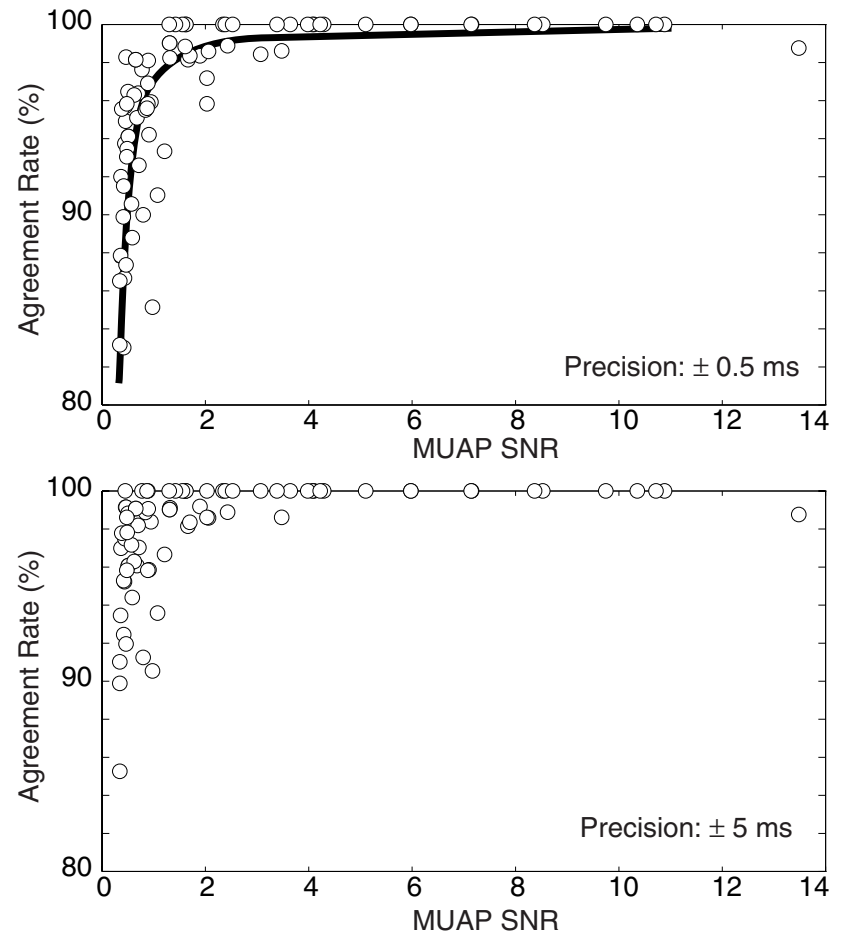

Figure 4. Agreement rates for all 83 pairs of MUAP trains. Each point corresponds to a pair of trains from the same MU that were decomposed independently in different signals. The percentage of discharges for which the two decompositions agreed to within \pm 0.5 (top) or $\pm 5 \mathrm{~ms}$ (bottom) is plotted against the smaller MUAP SNR. The solid curve can be used to estimate decomposition accuracy as a function of MUAP SNR.

missed MUAPs were rare because they had mostly been detected during decomposition due to the noticeable gaps they made in the firing patterns (such as MU 9 in Fig. 1).

\section{DISCUSSION}

Since EMG decomposition involves complicated and sometimes subjective analysis, it is important to demonstrate its accuracy in an objective and realistic way. The current study was based on cross-checking actual signals similar to those we analyze in our investigations. The only assumption made in this approach is that decomposition errors in separate signals will almost never be of precisely the same amount. The reasonableness of this assumption can be seen with respect to Fig. 2. Although both signals contain activity from the same MUs, the MUAPs in the two signals and the offsets between them are quite different. Thus if a particular configuration of A templates gives the best match to a particular segment of signal A, then that same configuration of B tem- 
plates (adjusted for conduction delays) is unlikely to give the best match to signal B unless it is in fact the correct configuration.

The results presented here demonstrate that EMGLAB can be quite accurate for decomposing EMG signals from low and moderate levels of contraction. This finding confirms the subjective impression of experienced operators that the occurrences of the larger MUAPs are almost always unambiguous, and that the occurrences of the smaller MUAPs are unambiguous most of the time and can be localized reasonably well the rest of the time by interpolating their firing patterns.

The most common type of error we encountered involved misalignment of small MUAPs within superimpositions of larger MUAPs. Large MUAPs often exhibit a variability from discharge to discharge that results in a residual noise that can obscure smaller MUAPs. In Fig. 2, the operator was able to infer from the firing patterns that there had to be an occurrence of MUAP 6 in signal A somewhere between 6.22 and 6.24 s. However, the superimposition-resolution algorithm did not find precisely the correct location because of the residual noise. Errors of this type were typically less than $5 \mathrm{~ms}$ in magnitude and had only a very small effect on the overall firing patterns (Fig. 3).

The curve in Fig. 4 can be used to estimate decomposition accuracy in other signals that have levels of complexity, background noise, discharge regularity, and MUAP variability comparable to ones in this study. For example, for MUAPs with peak amplitudes at least 2.5 times the rms signal amplitude, decomposition accuracy can be expected to be $98-100 \%$. For MUAPs with peak amplitudes less than the rms signal amplitude, decomposition accuracy can be expected to be less than $96 \%$. We suggest that decomposition results should always be accompanied by a frank estimate of their accuracy, based on an objective analysis such as the one presented here.

\section{REFERENCES}

[1] B. Mambrito and C. J. De Luca, "A technique for the detection, decomposition and analysis of the EMG signal," Electroencephalogr. Clin. Neurophysiol., vol. 58, pp. 175-88, 1984.

[2] C. J. De Luca, "Precision decomposition of EMG signals," Meth. Clin. Neurophysiol., vol. 4, pp. 1-28, 1993.

[3] D. Farina, A. Crosetti, and R. Merletti, "A model for the generation of synthetic intramuscular EMG signals to test decomposition algorithms," IEEE Trans. Biomed. Eng., vol. 48, pp. 66-77, 2001.

[4] Z. C. Lateva, K. C. McGill, and M. E. Johanson, "Electrophysiological evidence of adult human skeletal muscle fibres with multiple endplates and polyneuronal innervation," J. Physiol., vol. 544 (pt. 2), pp. 549-565, 2002.

[5] Z. C. Lateva and K. C. McGill, "Estimating motor-unit architectural properties by analyzing motor-unit action potential morphology," Clin. Neurophysiol., vol. 112, pp. 127-135, 2001.

[6] K. C. McGill, "Optimal resolution of superimposed action potentials," IEEE Trans. Biomed. Eng., vol. 49, pp. 640-650, 2002.

[7] D. Stashuk, "EMG signal decomposition: how can it be accomplished and used?"J. Electromyogr. Kinesiol., vol. 11, pp. 151-173, 2001. 